\title{
Complementary Medicine for Disease and IIIness Prevention? A Research Perspective
}

\section{E. Ernst}

Department of Complementary Medicine, School of Postgraduate Medicine and Health Sciences, University of Exeter

\section{Introduction}

Complementary medicine (CM) has been defined as 'diagnosis, treatment and/or prevention which complements mainstream medicine by contributing to a common whole, by satisfying a demand not met by orthodoxy or by diversifying the conceptual frameworks of medicine' [1]. A deliberate point is made in this definition to include the preventative aspect. It may turn out to be of growing importance to $\mathrm{CM}$. Many proponents of $\mathrm{CM}$ are keen to point out that, while mainstream medicine may be good at treatment of diseases, particularly acute conditions, CM's forte lies in the prevention of disease [e.g. 2]. Patients seem to have intuitively accepted this notion; a recent survey suggest that more than $50 \%$ of those Americans who use $\mathrm{CM}$ do so not to treat ailments but to remain healthy, i.e. to prevent disease and illness [3].

If one looks closer at the evidence for or against CM's role in disease prevention, one is stunned by the contrast of firmly held beliefs and the lack of reliable evidence to support them. This article will address some of these issues. It is not a systematic review of the existing data, but an attempt to briefly outline the complexity of the subject area.

\section{Which CM Modalities?}

Perhaps the most obvious complication of the question, 'Does CM prevent disease?' is the undeniable fact that so many different CM treatment modalities exist. Often one has little to do with the other, their only common denominator being that they are categorised as complementary. According to the above-mentioned survey [3], the four most prevalent treatments in the US are:

- relaxation techniques

- herbal medicine

- massage

- chiropractic.

But there are overt national differences. The four most popular therapies in the UK, for instance, seem to be:

- spinal manipulation (chiropractic and osteopathy)
- acupuncture

- homoeopathy

- medical herbalism.

This heterogeneity means that the preventative potential (or otherwise) has to be demonstrated for each therapy. In clinical practice more than one CM treatment modalities are often used in parallel, and many $\mathrm{CM}$ clinicians fear that by separating them out one might loose (some of) the benefit of the positively interacting whole. In research terms, this means that the entire treatment 'packages' have to be tested. This may be best achieved in pragmatic trials. Obviously such an approach would limit the interpretability of the eventual findings; in such 'black box' trials it is usually not possible to determine which element in the 'package' prevented which condition. If answers are required to this latter question, one would need to test single interventions after all.

\section{Which Diseases?}

The term 'prevention' covers all conditions known to mankind. This renders our question 'Does CM prevent disease?' impossible to answer in any meaningful way. Even if we have defined (as closely as possible) our therapeutic modality or our treatment 'package', it is obviously a different matter to investigate whether, for instance, individualised homoeopathy prevents common colds, asthma attacks or cancer.

In research terms, this adds a second dimension of complexity. It means that it is also essential to define the condition we aim to prevent. If the claim is that homoeopathy prevents $x$ different types of ailments, we would normally require $x$ separate sets of data in support.

\section{Morbidity or Mortality?}

Do we want to prevent heart attacks or death from heart attacks? These questions can be fundamentally different in terms of clinical strategy and are likely to require different research methodologies. Often the prevention of morbidity is an aim that is easier reached

E. Ernst, M. D., Ph. D, FRCP

Department of Complementary Medicine

School of Postgraduate Medicine and Health Sciences

University of Exeter

25 Victoria Park Road, Exeter EX2 4NT UK

Tel/Fax +441392424989, E-mail E.Ernst@ex.ac.uk 
and less problematic to research. The prevention of common colds, for instance, may not be all that difficult to achieve and certainly is relatively simple to investigate [e.g. 4, 5]. Yet very often in medicine we want to prolong life and not just prevent disease. In other words, we aim at preventing premature mortality. The concepts of preventing disease or premature death obviously overlap to a certain degree. Preventative CM, however, needs to define its precise goal and develop a clear focus for generating testable hypotheses which, in turn, require experimental testing.

\section{Primary, Secondary or Tertiary Prevention?}

A further dimension of complexity is brought about by the fact that prevention is an umbrella term for issues which are best separated. We ought to be clear whether we mean primary, secondary or tertiary disease prevention. Essentially primary prevention is targeted at totally healthy individuals. Its aim is to find out how these people can remain healthy for as long as possible. Secondary prevention deals with patients who already have developed signs of a given disease, e.g. angina pectoris as a symptom of coronary heart disease. Its aim is to determine how these patients can steer clear of major pathology, e.g. a myocardial infarction. Tertiary prevention is targeted at patients who are already affected by major disease. It aims at preventing further major events once a first event has occurred, e.g. preventing a second (possibly fatal) heart attack.

Again both the clinical and the research strategies will differ according to the target, i.e. primary, secondary or tertiary prevention. While primary prevention for coronary heart disease largely means elimination of risk factors (e.g. hypertension), tertiary prevention (in this setting) might entail risk factor control plus invasive treatments aimed at deblocking a coronary artery. CM's forte lies (by implication [e.g. 2]) in primary prevention. It is probably less relevant for secondary or tertiary prevention. In the absence of evidence, however, this is not much more than speculation.

\section{What Evidence Is Required?}

In principle, there are few differences between mainstream medicine and $\mathrm{CM}$ as to the type of evidence and the methodology by which it is produced [6]. The accepted gold standard for demonstrating efficacy or effectiveness in both cases is the randomised-controlled trial (RCT). What is true for treatment is essentially also true for prevention. The crucial difference, however, often lies in the feasibility of RCTs for preventative measures. RCTs to test preventive efficacy are possible for conditions which occur frequently in most peoples lives; for instance, trials to test the efficacy of Echinacea for the prevention of common cold are possible and do exist [5]. By contrast, RCTs are much more difficult, even impossible, for conditions that are less frequent, do not affect the majority of a population, or are likely to occur only in the relatively distant future, i.e. years or decades. Imagine an RCT in primary prevention of cancer. This trial would require thousands of (healthy) participants and treatment periods of many years to have enough power to detect statistically significant and clinically relevant effects. Even when we are dealing with primary prevention of a disease that affects nearly $100 \%$ of the population but takes decades to develop, e.g. arteriosclerosis, a primary prevention trial would be impossible to conduct largely for the same (logistic) reasons.

This does not meant that there are no other (so to speak 'second best') ways to evaluate the value of a given prophylactic intervention. If the Rolls Royce of conclusiveness is not available a VW might also take us to our destination. Various research tools might be helpful. One method widely used in mainstream epidemiological research is the case control study. With this design, one compares individuals who have a certain characteristic with others who lack this particular characteristic. Thus one can, for instance, determine whether healthy individuals who regularly consume Ginseng are less likely to develop cancer than those who do not [7]. Alternatively one can investigate individuals with and without a disease like cancer and might find that the former consume more onion and garlic than the latter [8]. This, in turn, might strengthen the hypothesis that the regular intake of Allium vegetables or ginseng prevents cancer $[7,8]$.

Unfortunately such conclusions are invariably burdened with a considerable degree of uncertainty. There could be many other differences between the two groups under comparison, and even in prospective case control studies it usually is not possible to account for all possible confounders. Therefore one can never be quite sure on the basis of this type of evidence about cause-effect relationships, e. g. was it really the ginseng consumption and not some undetermined (e.g. life style) variable that caused the lower cancer rates [7]?

A similar research strategy is to identify risk factors for a given disease through observational studies of large populations. The term 'risk factor' is often misused even by professionals who should know better: Risk factors are causally related to the disease in question. Many characteristics commonly termed 'risk factors' are, in fact, risk markers because causality has not been established beyond reasonable doubt.

A causal risk factor for peripheral arterial occlusive disease, (PAOD), for instance, is smoking. Thus smoking can be used as a target (the technical term is surrogate endpoint) for primary prevention studies of PAOD. If we were able to show that acupuncture helps people to stop smoking, this would indirectly render acupuncture an effective measure to prevent PAOD. The strategy is clear and, in the realm of mainstream medicine, has proved to be highly successful. Unfortunately this is not quite true for CM: Acupuncture, for instance, is not an efficacious measure for smoking cessation [9].

\section{Conclusion}

Many enthusiasts intuitively believe that CM's forte lies in the maintenance of good health, i.e. in prevention of disease. Unfortunately the subject is more complex than normally appreciated 
within $\mathrm{CM}$. Until we have convincing data, it is not possible to state with confidence that a given form of $\mathrm{CM}$ is effective in preventing a given condition. It follows firstly that we now should prepare to carry out the much needed (but difficult) research related to preventative CM. Secondly it is concluded that we should be cautious and abstain from overstating the largely unproven role of $\mathrm{CM}$ in the prevention of disease and illness.

\section{References}

1 Ernst E, Resch KL, Mills S, Hill R, Mitchell A, Willoughby M, White A: Complementary medicine - a definition. Br J Gen Pract 1995;45:506

2 Fulder S: Handbook of Complementary Medicine. Oxford, Oxford Univ Press, 1988.

3 Eisenberg D, David RB, Ettner SL, Appel S, Wilkey S, van Rompay M, Kessler RC: Trends in alternative medicine use in the United States, 1990-1997. JAMA 1998;280:1569-1575.

4 Ernst E, Pechol E, Wirz P: Regular sauna bathing prevents common colds. Ann Med. 1990; 22:225-227.
5 Melchart D, Walther E, Linde K, Brandmaier R, Lersch C: Echinacea root extracts for the prevention of upper respiratory tract infections. Arch Fam Med 1998;7:541-545.

6 Vickers A, Cassileth B, Ernst E, et al: How should we research unconventional therapies? A report from the Conference on Complementary and Alternative Medicine Research Methodology, National Institute of Health. Intern J Technol Assessment in Health Care 1997; 13:111-121.

7 Yun TK, Choi SY: Non-organ specific cancer pre- vention of ginseng: A prospective study in Korea. Int J Epidemiol 1998;27:359-364.

8 Ernst E: Can Allium vegetables prevent cancer? Phytomedicine 1997;4:79-83.

9 White AR, Rampes H: Acupuncture in smoking cessation; in Lancaster T, Silagy C (eds): Tobacco Addiction Module of The Cochrane Database of Systematic Reviews. Available in The Cochrane Library [database on disk and CD-ROM]. The Cochrane Collaboration, Issue 1, Oxford, Update Software, 1997. 